\title{
Modelica Library for Feed Drive Systems
}

\author{
Denis Özdemir Tobias Motschke Werner Herfs Christian Brecher \\ Laboratory for Machine Tools and Production Engineering (WZL) of RWTH Aachen University, Germany, \\ \{D.Oezdemir, T.Motschke, W.Herfs, C.Brecher\}@wzl.rwth-aachen.de
}

\begin{abstract}
As a part of machine tools and production machines, the primary task of feed drives is to create the contour of a workpiece by moving it and/or the tool along one or more axes according to the control input. This paper presents a Modelica library for feed drive systems consisting of electrical, electro-mechanical and mechanical components. The aim of the library is to provide engineers means to design feed drive models that can be parametrized with available data from component suppliers. The models are augmented with metrics and requirements to facilitate simulation analysis.
\end{abstract}

Keywords: Feed drives, servo motors, machine tools

\section{Introduction}

The design of feed drives systems is a complex technical problem due to the numerous requirements, design variables and interactions. Therefore, many computer-aided methods have been developed and simulation techniques are employed since the 1980s, e.g. (Simon 1986). These approaches mostly rely on signaloriented models that are well suited to describe control structures (Brecher 2002; Zirn 2008). However, using the signal-oriented approach for physical systems such as multi-mass oscillators yields complicated structures that are difficult to understand even for experts. An alternative is to couple control-simulation with multibody simulation or finite element software (Altintas et al. 2011). In industrial practice, however, these simulation approaches have hardly been applied as a recent survey shows (Brecher et al. 2014). While many machine suppliers see great potential in virtual prototypes, qualified personal and the costs of software are main obstacles.

In contrast to these simulation approaches motor sizing software such as Sizer by Siemens or Motion Analyzer by Rockwell Automation are widely used. These tools allow a quick selection of an adequate motor for a specified application, but the mechanical part of the system is assumed to be given and dynamic properties of the feed drive system as a whole are not taken into account.

The Modelica library for feed drive systems aims to close the gap between the elaborated simulation tech- niques on the one hand and the sizing software on the other hand. This means the library provides models that can be used with limited expert knowledge by leveraging the concept of component-orientation in Modelica. Such an approach implies models that can be parametrized from available supplier data. In addition to the behavioral equations, metrics and requirements are included in the components to highlight critical behavioral aspects.

The Modelica library for feed drive systems is part of a planned design environment for feed drive systems where an optimizer is used to find those parameters and components from a database that optimally fulfill the requirements. The concept of the design environment and its potential implementation in the design process of machine tools is presented in an additional paper (Özdemir et al. 2015). In a preceding paper parts of an earlier version of the library have been presented briefly (Herfs et al. 2015). This paper therefore focuses on the newly developed aspects.

The contents of this paper fall into three main parts. In Chapter 2 models of feed drives motors are described. Chapter 3 addresses mechanical components and Chapter 4 presents results from the simulation of the system as a whole. Finally, Chapter 5 summarizes the results and explains how these models are embedded into the feed drive design environment.

\section{Models for Feed Drive Motors}

Feed drive motors of modern machine tools are primarily permanent-magnet synchronous motors (PSM), which are therefore the focus of this paper (s. Chap. 2.1). The models for permanent-magnet synchronous motors with field weakening option (s. Chap. 2.2) as well as for linear motors (s. Chap. 2.3) can be easily developed based on the model of the PSM. Hereby, the object-oriented approach has the advantage that many components can be reused.

\subsection{Model for Permanently Excited Synchro- nous Motors (PSM)}

The Modelica Standard Library contains two models of the PSM in the Electrical.Machines and Magnetic.FundamentalWave sublibraries. The core of these PSM models is a model of the air gap, which describes 
the electromechanical torque as the cross product of current and magnetic flux in the dq-coordinate system that is fixed to the rotor (Kral, Haumer 2005; Kral 2011). While the PSM model in the Machines library uses space vectors for current, voltage and flux linkage the FundamentalWave model follows an even more physically rigorous approach with complex vectors. In the Machine library heat losses are considered with regards to the resistance of the stator windings and the damper cage. In addition eddy current losses in the stator core and mechanical losses depending on the speed are taken into account.

However, this rigorous physical approach requires a variety of physical parameters, e.g. the inductances in $\mathrm{d}$ - and q-direction, the leakage inductance of the stator and parameters for eddy current losses. Because these parameters are generally not available during drive selection, the PSM models of the Modelica Standard Library have to be simplified. Moreover, the standard PSM models do not include the design requirements that have to be considered during feed drive design.

\subsubsection{Behavior Model}

Typically available supplier data regarding the physical behavior is displayed in Table 1. Based on this data a standard model for the PSM (Schröder 2009, p. 394) can be implemented:

$$
\begin{aligned}
& U_{d}=-p \cdot \omega_{M} \cdot L_{D} \cdot I_{q}, \\
& U_{q}=L_{D} \cdot \frac{d I_{q}}{d t}+R \cdot I_{q}+p \cdot \omega_{M} \cdot \psi_{P M}, \\
& M_{M}=3 \cdot p \cdot \psi_{P M} \cdot I_{q}
\end{aligned}
$$

Here, $U_{d}, U_{q}$ and $I_{q}$ are the RMS values of the motor voltage and torque building current in the dqcoordinate system and $\omega_{M}$ denotes the angular velocity of the rotor, see Figure 1 . The number of pole pairs $p$, the effective inductivity $L_{D}$ and the winding resistance $R$ can be taken from the supplier data. The magnetic flux of the permanent-magnet $\psi_{P M}$ relates motor torque $M_{M}$ and torque-building current $I_{q}$ and

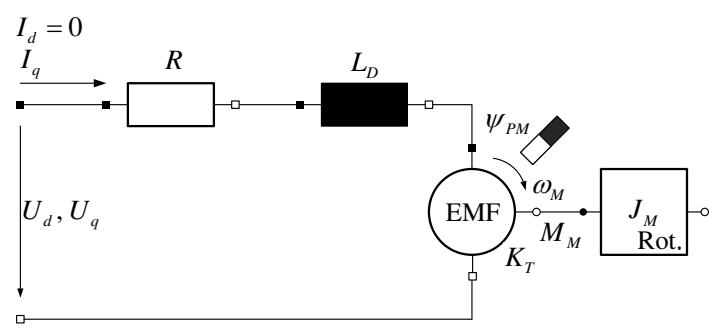

Figure 1: Equivalent circuit of the PSM can be calculated from the given torque constant $K_{T}$ by

$$
\psi_{P M}=\frac{1}{3 \cdot p} \cdot K_{T} .
$$

The temperature of the motor $T_{M}$ is dependent on the power dissipation $P_{V}$, the heat transfer resistance $R_{T h}$, the thermal time constant $t_{T h}$ and the environmental temperature $T_{U}$, i.e.

$R_{T h} \cdot P_{V}=t_{T h} \cdot \frac{d T_{M}}{d t}+\left(T_{M}-T_{U}\right)$.

While $t_{T h}$ is mostly listed in the supplier specification, there is usually no value for $R_{T h}$ given. But $R_{T h}$ can be calculated from the stall current at $100 \mathrm{~K}$ overtemperature $I_{0,100 K}$ :

$R_{t h}=\frac{100 K}{3 \cdot R \cdot\left(I_{0,100 K}\right)^{2}}$.

In addition to the winding resistance heat is dissipated due to iron losses and bearing friction at a rate approximately proportional to $\omega_{M}^{1,5}$. Overall the resulting motor losses are therefore

$$
P_{V}=3 \cdot R_{S t r} \cdot I_{A}^{2}+k_{R} \cdot\left|\omega_{M}\right|^{1,5},
$$

where $I_{A}$ denotes the effective value of the armature current that is equal to $I_{q}$ if no field weakening is applied. The proportionality factor $k_{R}$ for the iron and bearing friction losses can be estimated by the manufacturer's specifications of the motor current $I_{N, 100 K}$ for the rated torque $M_{N, 100 K}$ and the rated speed $\omega_{N}$, i.e.

$$
k_{R}=\frac{K_{T} \cdot I_{N, 100 K}-M_{N, 100 K}}{\sqrt{\omega_{N}}} .
$$

Table 1. Typically available physical data for a PSM

\begin{tabular}{lll}
\hline Variable & Unit & Physical parameter \\
\hline$p$ & - & Number of pole pairs \\
$K_{T}$ & $\mathrm{Nm} / \mathrm{A}$ & Torque constant \\
$K_{E}$ & $\mathrm{Vs} / \mathrm{rad}$ & Voltage constant at $20^{\circ} \mathrm{C}$ \\
$R$ & $\Omega$ & Winding resistance at $20^{\circ} \mathrm{C}$ \\
$L_{D}$ & $\mathrm{H}$ & Effective inductivity \\
$t_{\text {mech }}$ & $\mathrm{s}$ & Mechanical time constant \\
$t_{T h}$ & $\mathrm{~S}$ & Thermal time constant \\
$J_{M}$ & $\mathrm{~kg} \mathrm{~m}^{2}$ & Rotor inertia \\
\hline
\end{tabular}


The manufacturer specifications contain nominal values. However, some parameters can be determined more accurately when the actual state of the motor is taken into account. For example the resistance of the windings changes with temperature according to

$$
R^{*}=R \cdot\left(1+\alpha \cdot\left(T_{M}-293,15 \mathrm{~K}\right)\right)
$$

with $\alpha$ as the temperature coefficient of copper at $20{ }^{\circ} \mathrm{C}$. The torque constant $K_{T}$ is usually given for an overtemperature of $100 \mathrm{~K}$, i.e. $K_{T}=M_{0,100 \mathrm{~K}} / I_{0,100 \mathrm{~K}}$. Since the manufacturer data usually contains the stall torque and current also for $60 \mathrm{~K}$ overtemperature, a factor for the temperature dependence can be estimated by

$$
c_{k T, T}=1+\frac{K_{T}-M_{0,60 K} / I_{0,60 K}}{40 \mathrm{~K} \cdot K_{T}} \cdot\left(T_{M}-393 \mathrm{~K}\right) .
$$

Due to saturation effects at high currents, the torque constant decreases at torques $M_{M} \geq 2 \cdot M_{0,60 K}$ (Bosch Rexroth AG 2009; Siemens AG 2010). With the information on maximum torque and current the reduction factor $c_{k T, M}$ can be obtained by linear interpolation, so that

$$
K_{T}^{*}=c_{k T, T} \cdot c_{k T, M} \cdot K_{T} .
$$

\subsubsection{Requirement Model}

The requirement model of the PSM consists of the limit values and an adequate metric, see Table 2. For example, the line-to-line armature voltage $U_{A n}$ is limited by the maximum output voltage of the converter $U_{U, \max }$, i.e.

$$
\sqrt{3} \cdot U_{A n, \max } \leq U_{U, \max } .
$$

Substituting (2) and (3) into (12) yields for steady state

$$
\left(\frac{p \omega_{M} L_{D} M_{M}}{K_{T}^{*}}\right)^{2}+\left(\frac{M_{M} R^{*}}{K_{T}^{*}}+\frac{\omega_{M} K_{T}^{*}}{3}\right)^{2} \leq \frac{U_{U, \max }^{2}}{3},
$$

which allows to calculate the voltage limiting characteristic. These can then be compared to the torquespeed diagrams that are usually given by the motor supplier. Figure 2 shows a good correspondence between model and catalogue data regarding the voltage limiting characteristic; while Figure 3 shows the correspondence between the model and the manufacturers

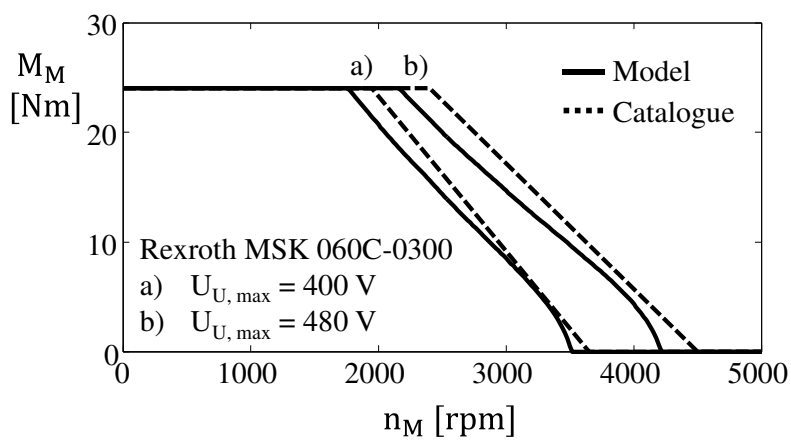

Figure 2: Comparison between calculated voltage limiting characteristics and supplier data sheet (Bosch Rexroth AG 2009)

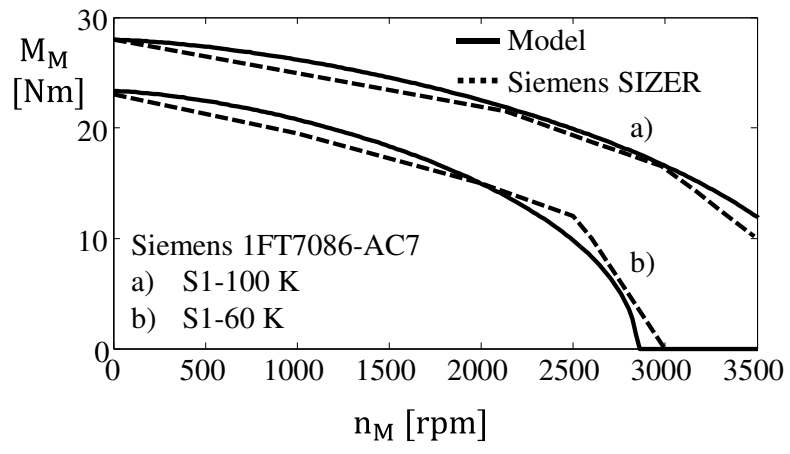

Figure 3: Comparison between calculated thermal limiting characteristics and supplier data sheet (Siemens AG 2010)

Table 2. Maximum permissible values for a PSM

\begin{tabular}{lll}
\hline Limit & Metric & Requirement \\
\hline DC link voltage & $U_{A n, \max }=\max _{t \in\left[t_{0}, t_{f}\right]}\left(\sqrt{\left(U_{d}(t)\right)^{2}+\left(U_{q}(t)\right)^{2}}\right)$ & $\sqrt{3} \cdot U_{A n, \max } \leq U_{U, \text { max }}$ \\
Temperature limit & $\Delta T_{M, \max }=\max _{t \in\left[t_{0}, t_{f}\right]}\left(T_{M}(t)-T_{U}\right)$ & $\Delta T_{M, \max } \leq \Delta T_{M, p e r m}$ \\
Current limit & $I_{A, \max }=\max _{t \in\left[t_{0}, t_{f}\right]}\left(\left|I_{A}(t)\right|\right)$ & $I_{A, \max } \leq I_{M, \max }$ \\
Torque limit & $M_{M, \max }=\max _{t \in\left[t_{0}, t_{f}\right]}\left(\left|M_{M}(t)\right|\right)$ & $M_{M, \max } \leq M_{M, I \text { max }}$ \\
Speed limit & $n_{M, \max }=\max _{t \in\left[t_{0}, t_{f}\right]}\left(\left|n_{M}(t)\right|\right)$ & $n_{M, \max } \leq n_{M, p e r m}$ \\
\hline
\end{tabular}


sizing tool. However, for other motors larger deviations are observed, see Figure 4. The deviations can be explained by parametric uncertainties and nonlinearities in the interpolation.

To validate compliance with the temperature limit, the actual motor temperature is obtained by combining (5) and (7). Here, the manufacturer usually indicates the so-called S1-Curve in the torque-speed diagram. Operation points below the S1-curve allow steady-state operation without violating the temperature limit.

\subsubsection{Modelica Model}

The behavioral equations and the requirements of the PSM are included in a Modelica model that can be parameterized with typical manufacturer data, see Figure 5. Equations (2)-(4) are modeled with the electrical equivalent circuit consisting of inverter, resistance, inductance, air gap, zero potential and sensors for voltage and current. Note, that not the connectors from the Modelica Standard Library are used since both effective values in the dq-coordinate system have to be con-

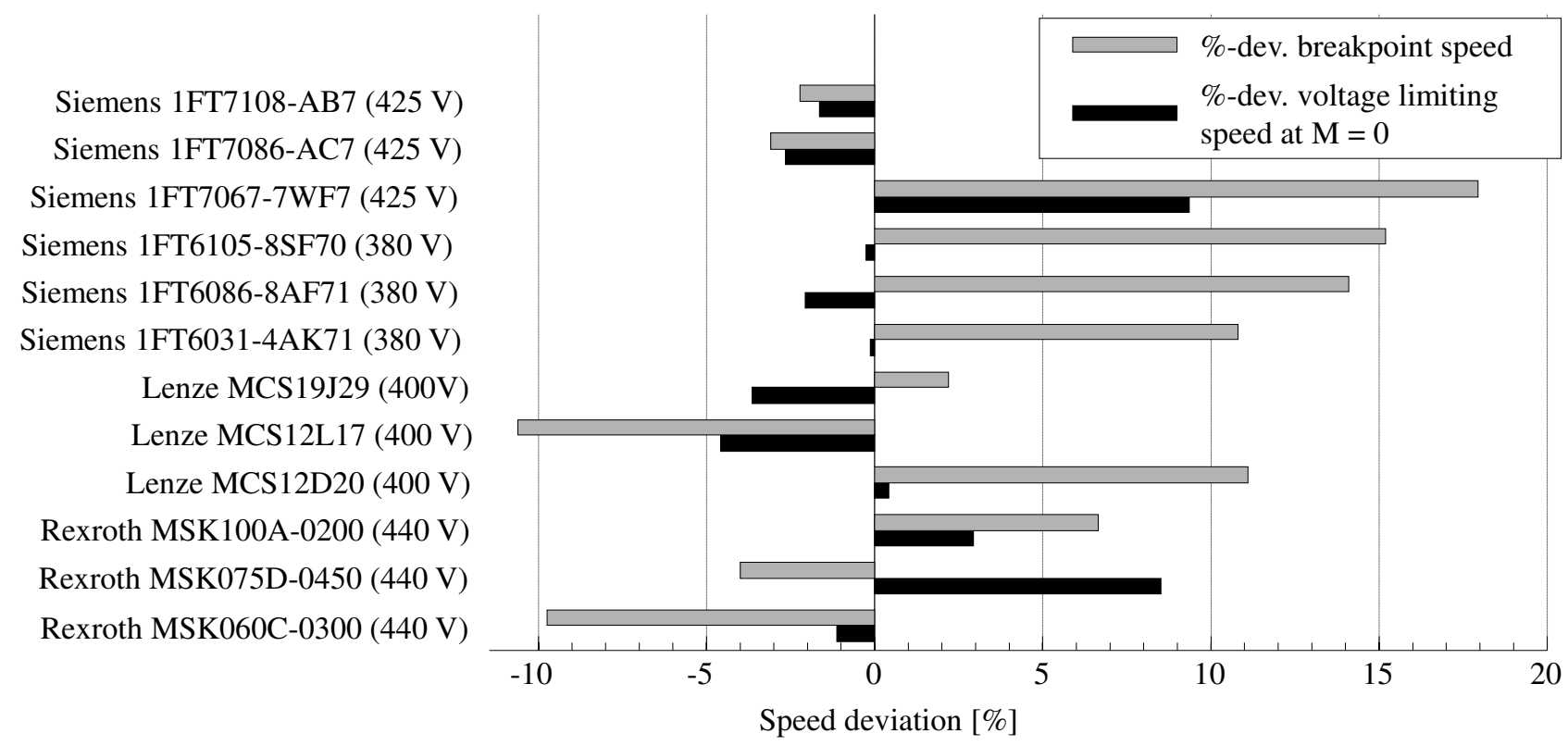

Figure 4: Percentage deviations of breakpoint speed and no-load voltage limiting speed for different motors

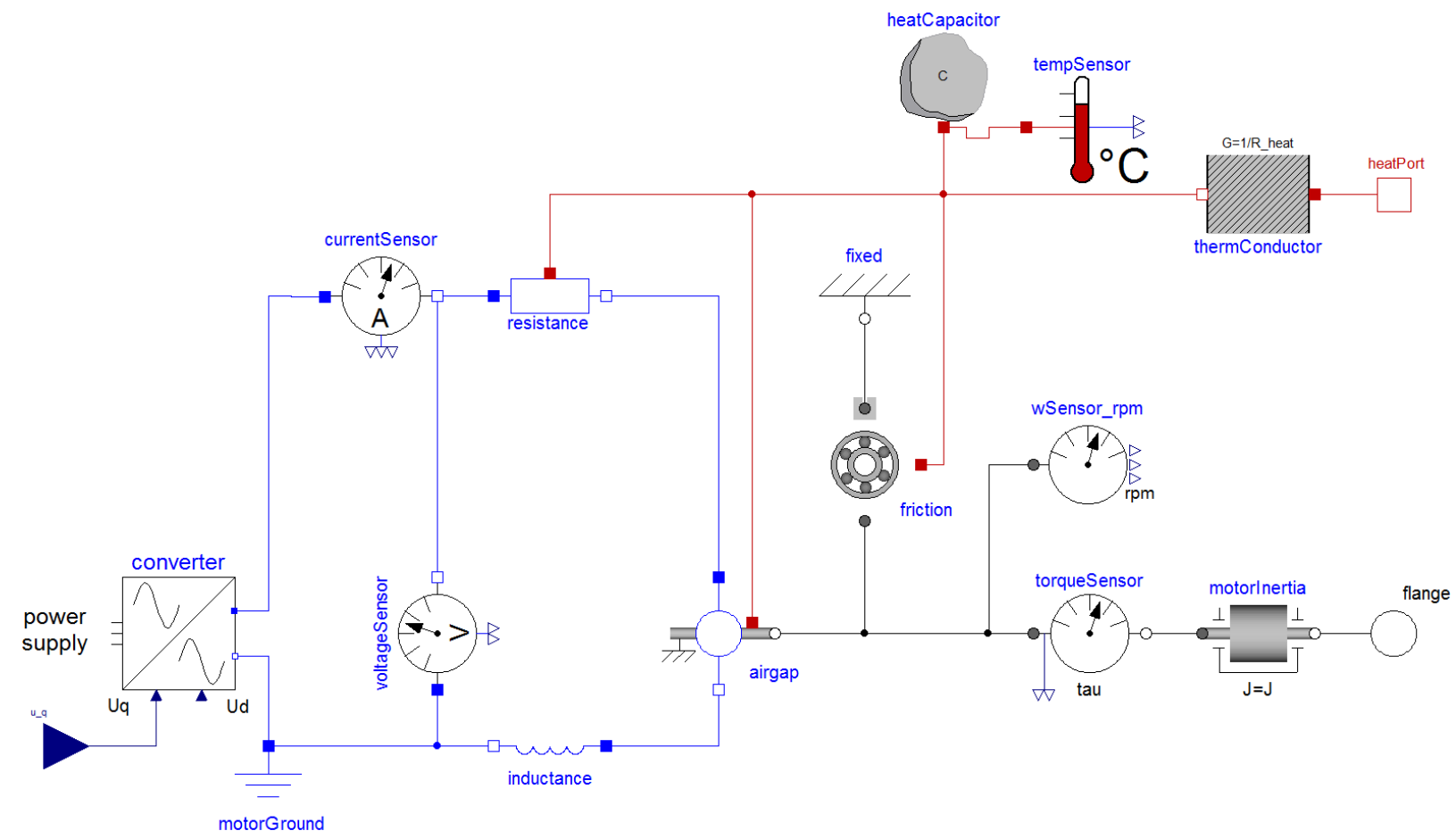

Figure 5: Permanently Excited Synchronous Motor in Modelica 
sidered. This means the connector contains voltage and current in d- and q-direction. In addition, the electrical connector includes a fifth variable for the electrical angular velocity. In contrast to the motor models from the Standard Library the three phase current and the harmonic oscillations are not explicitly simulated, since these are not required to describe the control behavior in the context of machine tools so that the simulation time can be shortened.

With the model of the converter the effective motor voltage in $\mathrm{d}$ - and q-coordinate direction can be impressed. The flux-generating current $I_{d}$ in the inverter is set to zero for the PSM without field weakening. The airgap model contains the relationship between torquebuilding current and torque (4) as well as between rotational speed and induced motor voltage (3). Moreover, the airgap includes the dependence of the torque constant on temperature and torque (11). The heat from the winding current and the iron and bearing friction losses yield the total heat loss that increases the temperature of the heat capacitor.

The metrics and requirements from Table 1 are included in the sensor models. For example, the current sensor contains the equations for maximum and RMS.

\subsubsection{Validation of the Current Control Loop Model}

According to (3), the relationship between torquebuilding current $I_{q}$ and effective motor voltage in qcoordinate direction $U_{q}$ can be described with a first order time lag. $I_{q}$ is controlled with a PI controller and the electrical time constant is compensated by tuning the PI controller with the magnitude optimum criterion, see Figure 6 (left). The step response is measured for a servomotor of type Siemens 1FT6108 and compared to simulation, see Figure 6 (right). The settling time of simulation and measurement is similar, but the measured trajectory of $I_{q}$ oscillates at a lower frequency. The frequency response - that has been obtained with Modelica Linear Systems Library - underlines this difference in the dynamic behavior since the maximum elevation of the simulation occurs at just under $200 \mathrm{~Hz}$ while the measured maximum lies at $600 \mathrm{~Hz}$, see Figure 7. The amplitude response reflects the delays of the electrical system and the measurement system with a drop of $40 \mathrm{~dB}$ per decade. The differences between measurement and simulation are probably due to the simplified control structure in the model. Regarding the outer control loops in the cascaded feed drive structure, the difference can be neglected.
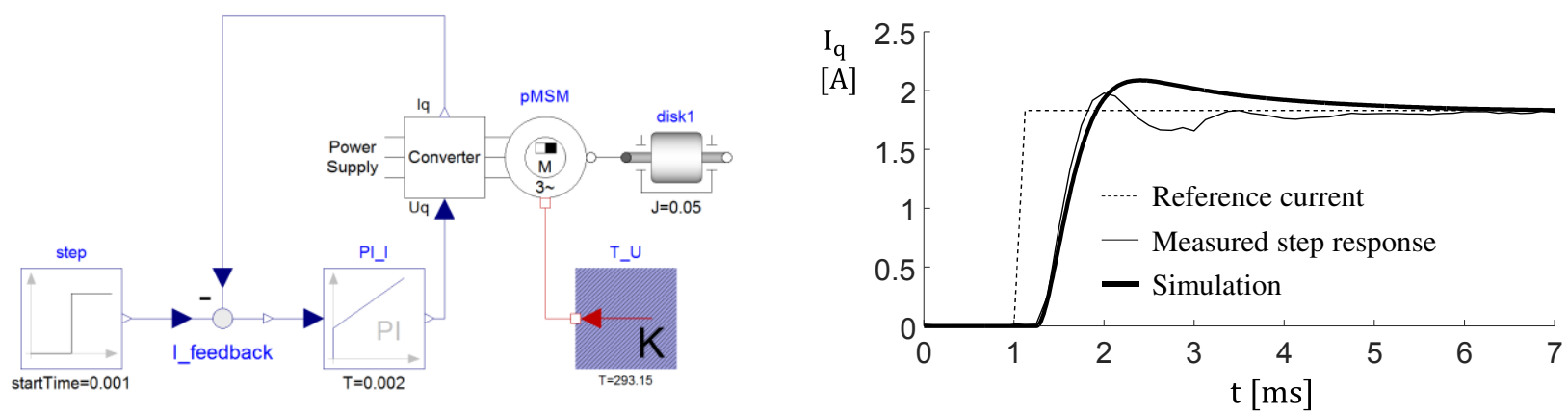

Figure 6: Model of the current control loop (left) and step response (right)
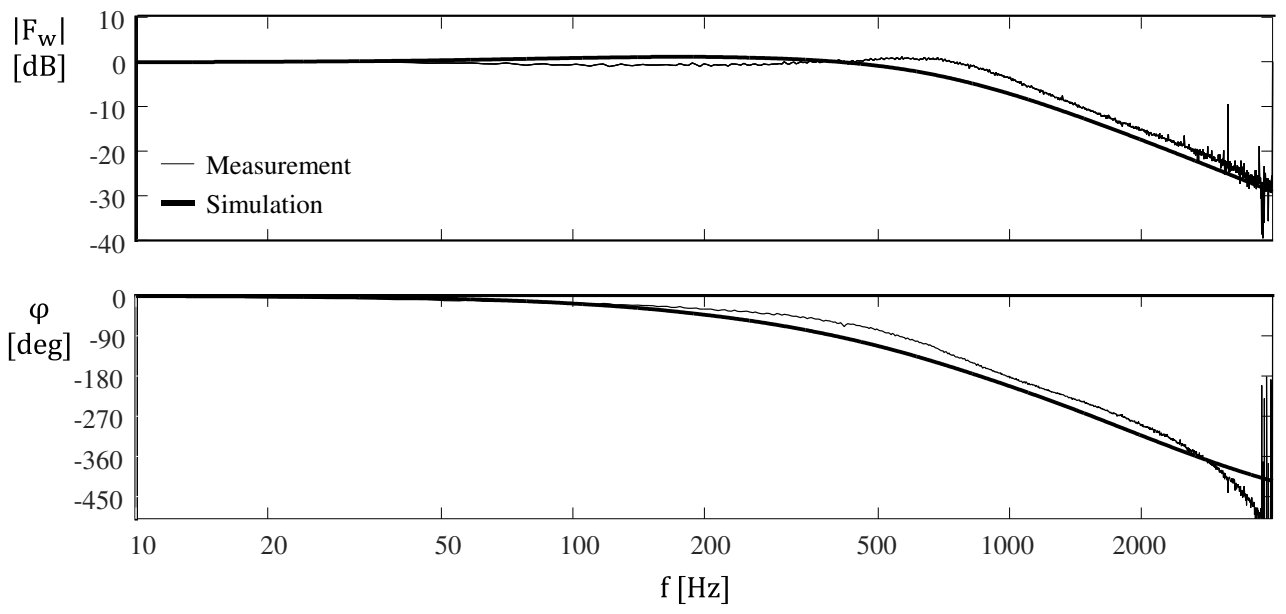

Figure 7: Reference frequency response of the current control loop 


\subsection{Model for Permanently Excited Synchro- nous Motor with Field Weakening Option (PSM-FW)}

The aim of field weakening is to expand the possible operating range of the motor by eliminating the restriction of the voltage limiting curve. For this purpose a counter voltage is induced that weakens the magnetic field. For the PSM, field weakening can only be achieved by a current component in the d-coordinate axis that compensates the magnetic field of the permanent-magnet. Given the possibility of field weakening, but neglecting reluctance, (3) is extended to (Schröder 2009, p. 391):

$$
U_{q}=L_{D} \frac{d I_{q}}{d t}+R^{*} \cdot I_{q}+p \cdot \omega_{M}\left(\psi_{P M}+L_{D} \cdot I_{d}\right)
$$

which illustrates that a negative current $I_{d}$ reduces voltage component $U_{q}$. On the one hand, the new limiting curve results from the condition $\sqrt{I_{d}^{2}+I_{q}^{2}} \leq I_{M, \max }$. On the other hand, there is a new voltage limit at the point of maximum field weakening at $I_{d}=-\psi_{P M} / L_{D}$. In this case the product of rotational speed and torque is constant, i.e. the points on the voltage characteristic with field weakening correspond approximately to the same power.

From a modelling point of view, field weakening can be described as follows. If the converter output voltage without field weakening $U_{A, \neg F W}$ is below the permissible value, no field weakening is required. Once the converter output voltage without field weakening would exceed the permissible value, a negative current in d-coordinate direction is induced that maintains the voltage $U_{A}=\sqrt{3} \cdot \sqrt{U_{d}^{2}+U_{q}^{2}}$ below the permissible value. Therefore, there are two alternative system equations:

$$
\left\{\begin{array}{ll}
I_{d}=0, & \text { if } U_{A, \neg F S} \cdot S_{U} \leq U_{U, \max } \\
U_{A} \cdot S_{U}=U_{U, \max }, & \text { if } U_{A, \neg F S} \cdot S_{U}>U_{U, \max }
\end{array},\right.
$$

where $S_{U}$ denotes a safety factor that determines how far the voltage is kept below the limit. In an exemplary case a motor is operated at a point just below the limiting curve of field weakening, see Figure 8 (left). The armature voltage increases with the speed up to the specified limit of $U_{U, \text { max }} / S_{U}=400 \mathrm{~V}$, see Figure 8 (right). Once the limit is reached, $I_{d}$ keeps the voltage constant. The peak of $I_{d}$ is just below the limit value of $-I_{d}=\psi_{P M} / L_{D}=20 \mathrm{~A}$ which corresponds to the operating point just below the field-weakening curve. It should be noted that an implementation according to (15) has the advantage that no controller tuning is required. However, reaching the maximum of the field weakening current leads to termination of the simulation since the algebraic equation system can no longer be resolved.

\subsection{Model for Linear Motors}

The percentage of linear induction machines as drive systems in machine tools is generally estimated below $10 \%$. Nevertheless it has its advantages in high dynamics and the lack of transmission elements. A disadvantage is the small accessible feed force in relation to the costs. The model of the linear motor follows from the PSM model by transferring rotational to translational dimensions, i.e. instead of $\omega_{M}$ we have translational velocity $v_{M}$, instead of $p$ the pole pitch $\tau_{p}$, and instead of $K_{T}$ the force constant $K_{F}$. This yields:

$$
\begin{aligned}
& U_{d}=-\frac{v_{M} \cdot \pi}{\tau_{p}} \cdot L_{D} \cdot I_{q}, \\
& U_{q}=L_{D} \cdot \frac{d I_{q}}{d t}+R^{*} \cdot I_{q}+\frac{K_{F}^{*}}{3} \cdot v_{M}, \\
& F_{M}=K_{F}^{*} \cdot I_{q} .
\end{aligned}
$$
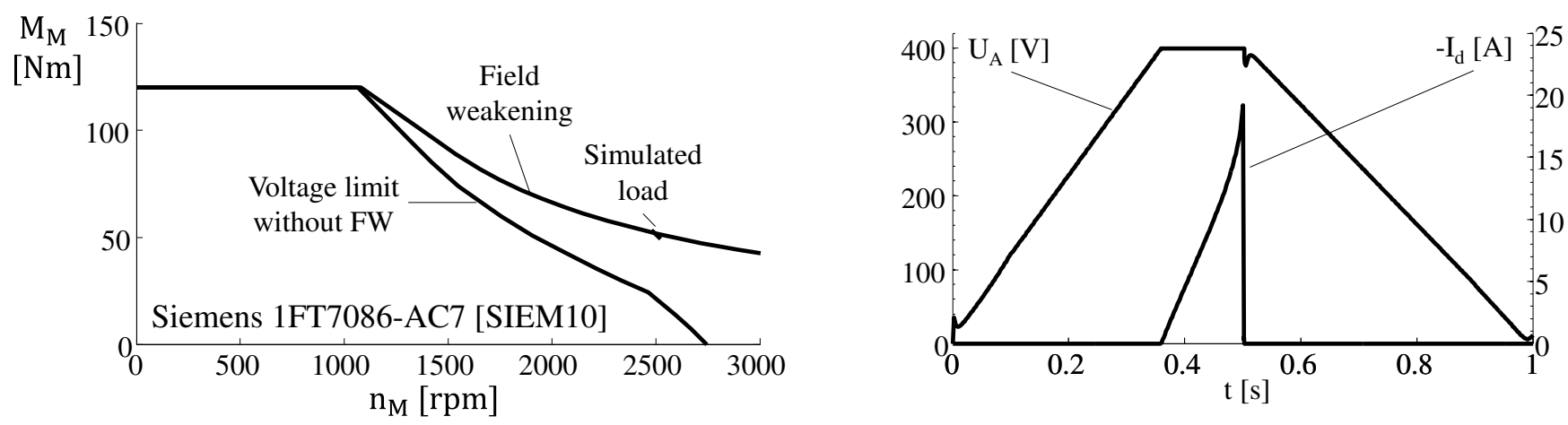

Figure 8: Operation points in the torque-speed-characteristic of the supplier (1), simulated trajectories for armature voltage and field-weakening current (r) 
In terms of the thermal behavior, modeling of the linear motor varies from the PSM. Due to its structure a cooling system is deployed. The cooling cycle is described by

$T_{R}=T_{M}+\left(T_{V}-T_{M}\right) \cdot \exp \left(-\frac{1}{R_{t h} \cdot \dot{V} \cdot \rho \cdot c_{p}}\right)$,

where $T_{R}$ is the recirculation temperature, $T_{V}$ the forward flow temperature, $R_{t h}$ the thermal resistance, $\dot{V}$ the volume flow, $\rho$ the fluid density and $c_{p}$ the spe- cific heat capacity of the fluid. The pressure drop is obtained from the formula of Blasius

$$
\Delta p=K_{p} \cdot \dot{V}^{1,75}
$$

where the factor $K_{p}$ can be calculated from nominal flow rate and pressure drop as given in the manufacturer data. Figure 9 shows the linear induction motor model. The similarity to the rotational induction machine is obvious. The rotational parts such as rotor, rotational sensor and electromagnetic force have been

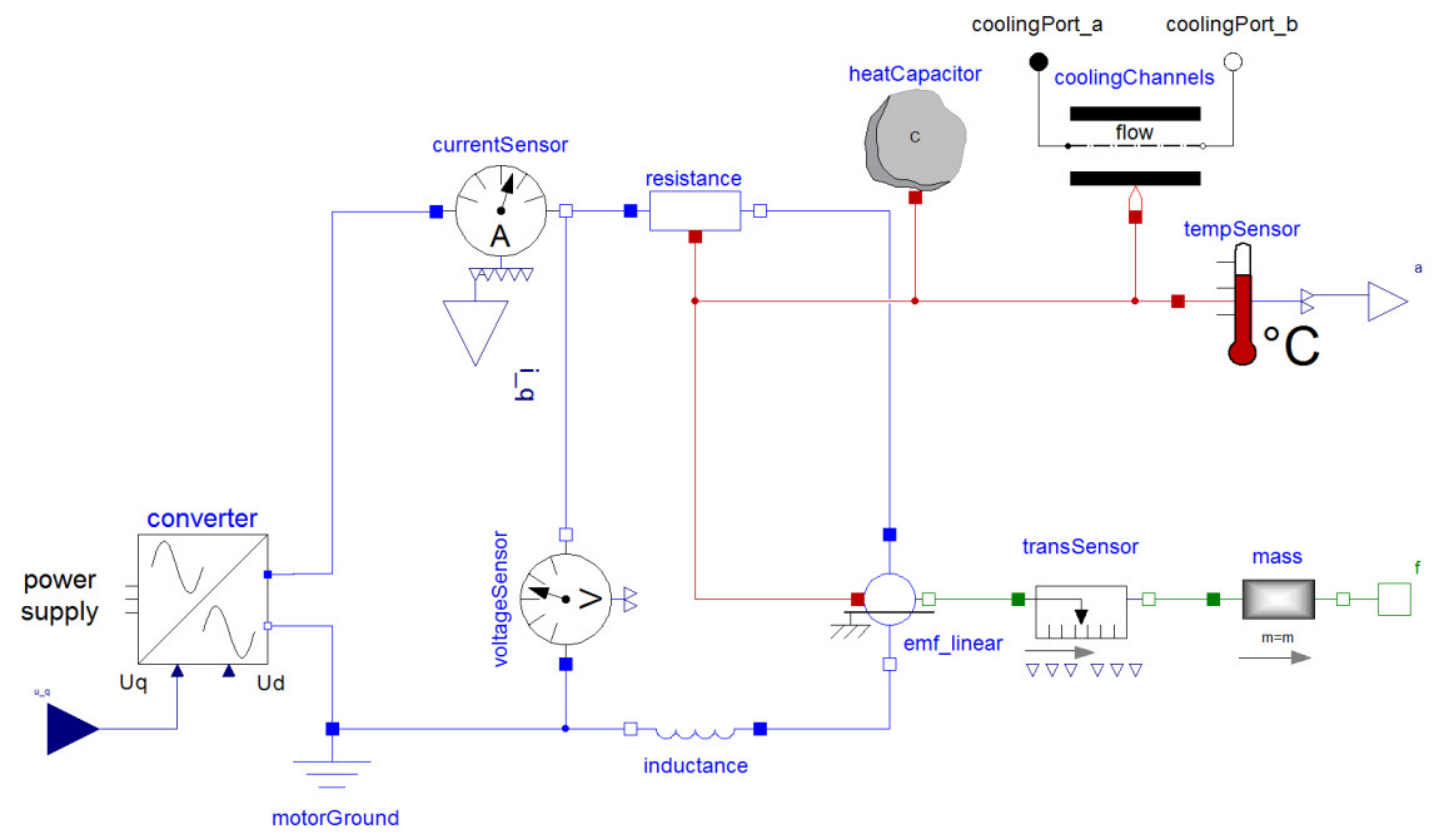

Figure 9: Modelica model for linear motors in Modelica

Table 3. Maximum permissible values for ball screw drives based on (Gross et al. 2006)

\begin{tabular}{lll}
\hline Limit & Metric & Requirement \\
\hline Eigenfrequency & $f_{d}=\frac{1}{2 \pi} \sqrt{\frac{1 / m_{r e f}}{1 / c_{M}+l_{S p} /\left(c_{S p}^{\prime} \cdot k_{S p, L}\right)}}$ & $f_{d} \geq f_{d, \text { min }}$ \\
Strain to preload & $F_{S p, \max }=\max _{\left.t \in t_{0}, t_{f}\right]}\left(\left|F_{S p}(t)\right|\right)$ & $F_{S p, \max } \leq 2^{3 / 2} \cdot F_{a, V M}$ \\
Strain to collapse load & $F_{S p, \max }=\max _{\left.t \in t_{0}, t_{f}\right]}\left(\left|F_{S p}(t)\right|\right)$ & $S_{K n} \cdot F_{S p, \max } \leq k_{S p, K n} \cdot \frac{d_{S p}{ }^{4}}{l_{S p}{ }^{2}}$ \\
Strain to static rating & $F_{S p, \max }=\max _{\left.t \in t_{0}, t_{f}\right]}\left(\left|F_{S p}(t)\right|\right)$ & $S_{0 a m} \cdot F_{S p, \max } \leq C_{0 a m}$ \\
Critical bending speed & $n_{S p, \max }=\max _{t \in\left[t_{0}, t_{f}\right]}\left(\left|n_{S p}(t)\right|\right)$ & $S_{n} \cdot n_{S p, \max } \leq k_{S p, n} \cdot \frac{d_{S p}}{l_{S p}{ }^{2}}$ \\
DN-Value & $n_{S p, \max }=\max _{t \in\left[t_{0}, t_{f}\right]}\left(\left|n_{S p}(t)\right|\right)$ & $n_{S p, \max } \leq D N_{p e r m} / d_{S p}$ \\
Lifetime & $\frac{2 \cdot\left(C_{a m} / F_{m a}\right)^{3} \cdot 10^{6}}{n_{m} / \min ^{-1} \cdot 60}$ & $L_{h} \geq L_{h, \min }$
\end{tabular}


replaced by translational components. The electrical parts e.g. inverter and stator remain the same.

\section{Mechanical Transmission Elements}

Beside the servomotor, feed drives require transmission elements to transfer forces and moments from drive to tool. These elements also need a proper model to determine their influence on the drive system. Realistic component models can help predicting lifetime and strain limits of components. Taking inherent or geometric restrictions into account during simulation, the search for optimal system components is facilitated.

In the following the approach will be demonstrated by the example of a ball screw drive. Ball screw drives are the most frequently used feed elements for machine tools up to $4 \mathrm{~m}$ traveling distance to realize the transmission from rotational to translational motion. The translational motion is characterized by the spindle pitch $h_{S p}$ and the relation between translational and rotation velocity is given by

$$
\frac{v}{\omega}=\frac{h_{S p}}{2 \pi},
$$

Spindle torque $M_{S p}$ and force $F_{S p}$ are described by a regular mechanical equation of motion

$$
M_{S p}=J_{S p} \cdot \dot{\omega}_{S p}+F_{S p} \cdot \frac{h_{S p}}{2 \pi}+\sum M_{F},
$$

where $\sum M_{F}$ sums up the losses of the ball screw nut and the force dependent friction of the spindle bearings. The model contains the components spindle shaft, spindle bearing, spindle inertia and ball screw nut. The model for the screw nut defines the restrictions according to eigenfrequency, maximum strain, revolution limit, lifetime and the lossy transmission from rotational to translational movement. The corresponding requirements in Table 3 are formulated similarly to those of the PSM.

\section{Feed Drive System Simulation}

A major advantage of using object-orientated models for feed drives is that modelling and simulation is possible with limited expert knowledge if an adequate component library is available. Once the system topology is known, the component models from the library can be connected and parametrized by the user from available data of catalogues. As an example for linked models one axis of a machine tool feed drive is shown in Figure 10. The design is parametrized by characteristic values that are available at an early design stage like stiffness of motor, clutch, spindle, spindle nut or bearings. Another advantage of the objective oriented approach is the intuitive connection of components. While signal oriented modeling demands mathematical knowledge regarding transfer from the user, the objectoriented approach allows to maintain the physical topology. In addition to the motor and the mechanical components Figure 10 shows the control loops. Using the Linear Systems Library one is able to determine the frequency response functions of the system, see Figure 11. As expected the correspondence between simulation and experiment shows differences in the dynamic behavior. This circumstance is owed to the simple model neglecting several compliances of the complex mechanical system. Nevertheless the dominant resonance at approximately $350 \mathrm{~Hz}$ is reproduced well by the model that only contains a-priori data. The simulation results show that with small modeling effort and a-

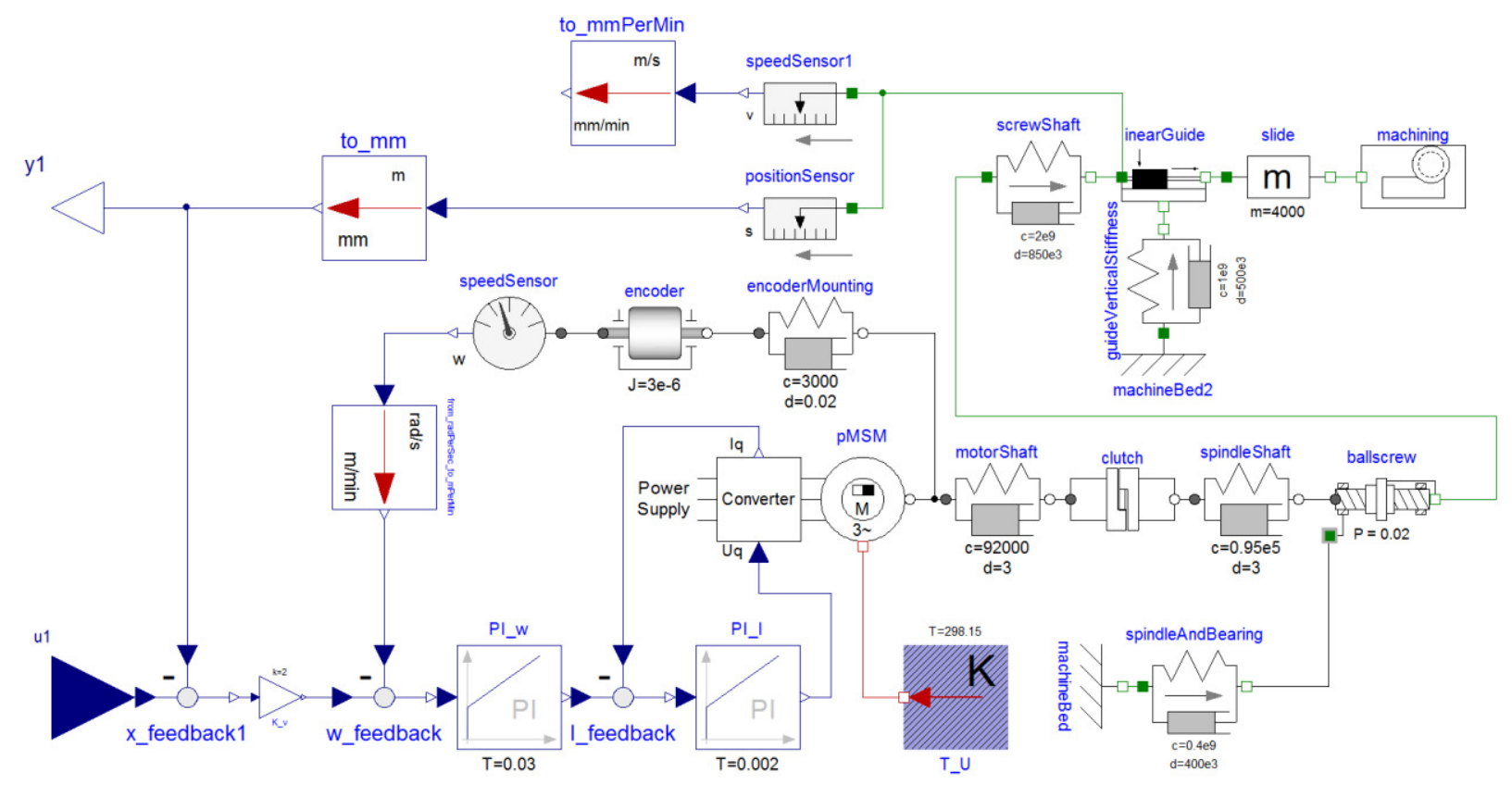

Figure 10: Drive topology for on axis of a machining center 
priori available component data a prediction of the dynamic behavior and critical operation states is possible to some extent. For a detailed structural analysis, however, a FEM-simulation is necessary. Linked to a component database a sensitivity analysis can be deployed to find more suitable components in terms of the desired system properties like efficiency or other performance defining quantities.
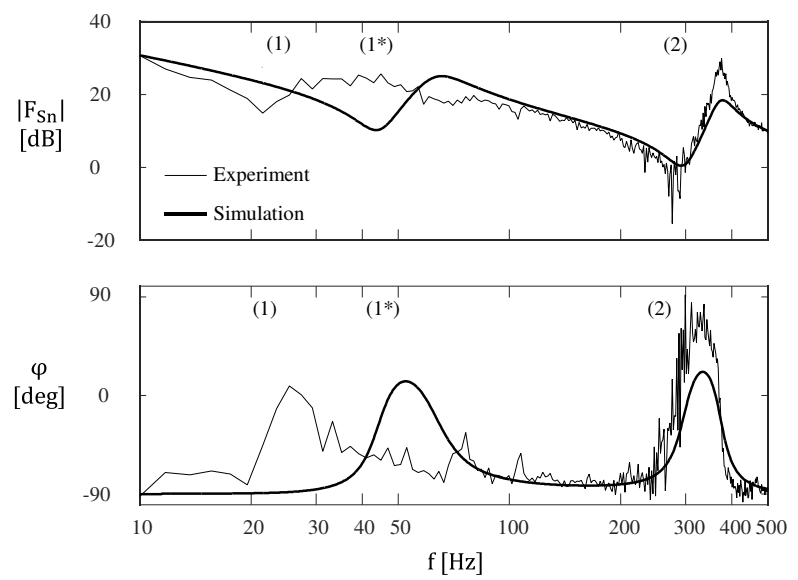

Figure 11: Correspondence between experimental and simulated frequency response

\section{Conclusion and Outlook}

This paper presented the Modelica library for feed drives. The behavior equations are based on wellknown equations. It has been shown, how these equations can be incorporated in a model that can be parametrized with a-priori available data. Moreover, the paper outlines, how design requirements can be included in the component models. The library will be submitted to the Modelica Association as a free library by the end of 2015.

The final goal is to parametrize the object-oriented model so that it complies with the system specific restrictions such as limited dc voltage supply, temperature or energy consumption. The variables of the model serve as input for the linked optimization tool. Then the parameters of existing products, obtained from supplier catalogues for instance, are implemented in a database that is directly connected to the model library of the simulation tool. During an optimization run the optimizer has access to the database and is able to vary the model parameters at every iteration step. The optimization algorithm is therefore responsible for the systematic search for the best solution, without simulating the whole solution space that is containing all possible combinations of components. Beside the optimization of component parameters, it is also possible to analyze the system dynamics and to optimize the control system. By defining characteristic parameters for the dynamic behavior such as step response or frequency response as target functions, the controllers are predesigned directly during the engineering stage of the development process.

\section{Acknowledgement}

The authors thank the German Research Foundation DFG for the support within the project "Optimierung des Systementwurfs von Maschinen und Anlagen auf Basis komponentenorientierter Verhaltensmodelle".

\section{References}

Altintas, Y.; Verl, A.; Brecher, C.; Uriarte, L.; Pritschow, G. (2011): Machine tool feed drives. In CIRP Annals - Manufacturing Technology 60 (2), pp. 779-796. DOI: 10.1016/j.cirp.2011.05.010.

Bosch Rexroth AG (2009): Rexroth IndraDyn S. Synchronmotoren MSK.

Brecher, Christian (2002): Vergleichende Analyse von Vorschubantrieben für Werkzeugmaschinen. RWTH Aachen: Dissertation.

Brecher, Christian; Brockmann, Birk; Daniels, Matthias; Wennemer, Matthias (2014): Herausforderungen bei der messtechnischen Untersuchung von Werkzeugmaschinen. In Zeitschr.f. wirtsch. Fabrikbetrieb (12), pp. 885-888.

Gross, Hans; Hamann, Jens; Wiegärtner, Georg (2006): Technik elektrischer Vorschubantriebe in der Fertigungsund Automatisierungstechnik. Erlagen: Publicis.

Herfs, W.; Özdemir, D.; Lohse, W.; Brecher, C. (2015): Design of Feed Drives with Object-Oriented Behavior Models. In Inge Troch, Andreas Kugi, Felix Breitenecker (Eds.): MATHMOD 2015 - 8th IFAC International Conference on Mathematical Modelling, Vienna, pp. preprint.

Kral, C.; Haumer, A. (2005): Modelica libraries for dc machines, three phase and polyphase machines. In Proc. of the 4th Modelica Conference, pp. 549-558.

Kral, C.; Haumer, A. (2011): The New FundamentalWave Library for Modeling Rotationg Electrical Three Phase Machines. In: Proc- of the $8^{\text {th }}$ Modelica Conference, pp. 170-179

Özdemir, D.; Herfs, W.; Brecher, C. (2015): Approaching the Dilemma between Plan and Value in Computer Aided Engineering of Production Machines. In Proc. of the 48th CIRP Conference on Manufacturing Systems, Ischia accepted.

Schröder, Dierk (2009): Elektrische Antriebe - Grundlagen. $4^{\text {th }}$ ed. Dordrecht: Springer.

Siemens AG (2010): SINAMICS S120. Synchronmotoren 1FT7. Projektierungshandbuch 03/2010. Erlangen: Siemens AG.

Simon, Walter (1986): Elektronische Vorschubantriebe an NC-Systemen. Technische Universität München: Dissertation.

Zirn, Oliver (2008): Machine Tool Analysis. Modelling, Simulation and control of Machine Tool Manipulators. ETH Zürich: Habilitation. 